\title{
Die Brüder Ferdinand III. und Leopold Wilhelm, ihre Stellung zu ihrem Lehrer Giovanni Valentini, zur Dichtung und zur Musik
}

\section{The Brothers Ferdinand III and Leopold Wilhelm, Their Attitude Towards Their Teacher Giovanni Valentini and Towards Poetry and Music}

Herbert Seifert / herbert.seifert@univie.ac.at

Institute of Musicology, University of Vienna, AT

\begin{abstract}
The brothers Ferdinand III and Archduke Leopold Wilhelm shared their interests in poetry and music since their youth. Both of them wrote poems, but only Ferdinand composed music. He had his own musical establishment since 1630, but his younger brother, a cleric and military leader, did not care about one before 1640/41, when he sent the composer Giacinto Cornacchioli to Italy to hire musicians and a poet and to buy music. When he arrived at Brussels as governor in 1647, he enlarged this establishment and had the first opera performed there in 1650. The letters by Cornacchioli and the brothers are the main sources for the new informations we now possess. In two short digressions other findings are proposed: the reason for Giovanni Priuli's leave from the Imperial court in 1626 and the retrieval of the 17th-centuryscore of Ferdinand III's opera "Drama musicum" (1648), missed since 1893.
\end{abstract}

\section{Keywords}

Emperor Ferdinand III, Archduke Leopold Wilhelm, poetry, Hofkapelle, Brussels, Pietro Verdina, Giacinto Cornacchioli, Gioseffo Zamponi, opera, Giovanni Priuli 
Der zunächst in Graz residierende Erzherzog und spätere Kaiser Ferdinand II. hatte zwei Söhne. Der ältere, ebenfalls Ferdinand getauft, wurde 1608 in Graz geboren, der jüngere, Leopold Wilhelm, Anfang 1614 in Wiener Neustadt. Sie folgten ihrem nun kaiserlichen Vater nicht schon 1619 in die neue Residenz Wien, sondern übersiedelten erst 1624 aus Graz dorthin. ${ }^{1}$ Im November 1627 wurden in Prag die Kaiserin zur Königin und Erzherzog Ferdinand zum König von Böhmen gekrönt. Dieser berichtete seinem in Wien gebliebenen Bruder Leopold Wilhelm in lateinischen Briefen unter anderem über die Aufführungen einer italienischen Komödiantentruppe, über die von den Hofmusikern gespielte Oper La Transformatione di Calisto und über ein Ballett. ${ }^{2}$ Einem Brief vom Jänner 1628 legte der 19jährige König von Böhmen sogar ein eigenes italienisches Gedicht bei. ${ }^{3}$

In der Folgezeit zeigte sich, dass beide Brüder eine literarische Ader hatten und in der am Kaiserhof für Theater und Dichtung bevorzugten italienischen Sprache dichteten, dass der Kronprinz und ab 1637 Kaiser Ferdinand III. aber zusätzlich noch komponierte. Über diesen schrieb der venezianische Botschafter Sebastian Venier schon 1630 aus Wien, dass er Verse und Musik sehr gut verfasse ${ }^{4}$.

In einem Brief Ferdinands aus Regensburg vom 20. Juli 1630 an seinen Bruder findet sich der Nachweis dafür, denn er nennt italienische Verse, die er auch in Musik gesetzt habe $^{5}$. Als er im Mai 1636 wieder in dieser süddeutschen Stadt weilte, um zum Römischen König gekürt zu werden, schrieb er von seiner Komposition für zwei Tenöre, die er dort aufführen lassen wollte. ${ }^{6}$ Ihr folgten noch zahlreiche lateinische liturgische Werke, italienische Madrigale und Canzonetten und sogar eine italienische Oper mit allegorisch-moralischer Handlung, die er 1649 an den gelehrten Jesuiten Athanasius Kircher nach Rom schickte ${ }^{7}$. Von diesem hatte er im selben Jahr ein „Komponierkästchen“ erhalten, das sein nach Rom gesandter Organist Johann Jacob Froberger mitgebracht hatte, einen Behelf, den er offenbar gleich für viele Kirchenwerke verwendete. Und im folgenden Jahr erschien Kirchers umfassende Musurgia universalis mit einer Widmung an Leopold Wilhelm und einem seiner Madrigaltexte, vom Kaiser in Musik gesetzt, „Chi volge nella mente“. Der Erzherzog ließ dem Verfasser dafür 1500 Gulden überwei-

1 SCHREIBER, Renate. "Ein Galeria nach meinem Humor". Erzherzog Leopold Wilhelm. Wien: Kunsthistorisches Museum 2004, S. 14.

2 SEIFERT, Herbert. Die Oper am Wiener Kaiserhof im 17. Jahrhundert (Wiener Veröffentlichungen zur Musikwissenschaft 25). Tutzing: Schneider, 1985, S. 596, 598, 601.

3 ANTONICEK, Theophil. Musik und italienische Poesie am Hofe Kaiser Ferdinands III. (Mitteilungen der Kommission für Musikforschung 42). Wien: Verlag der Österreichischen Akademie der Wissenschaften, 1990, S. 3.

4 SAUNDERS, Steven. The Emperor as Artist. New Discoveries Concerning Ferdinand III's Musical Compositions In Studien zur Musikwissenschaft 45, 1996, S. 7-31: 27.

5 Wien, Haus-, Hof- und Staatsarchiv, Habsburgisch-lothringisches Familienarchiv (Hausarchiv), Familienarchiv A, Karton 11, fol. 27r: Das Gedicht hat den Titel Occhi sdegnosi, besteht aus vier Strophen und beginnt mit dem Vers „O spietatissimi“. Seine nicht erhaltene Vertonung hatte Ferdinand am 14. Juli 1630 aufführen lassen.

6 ANTONICEK., op. cit., S. 6.

7 s. unten Exkurs 1. 
sen und erhielt 50 Exemplare des gewichtigen Werks, ebenso viele sein Bruder Ferdinand III ${ }^{8}$.

Dieser hatte als Kronprinz schon seit spätestens 1630 eine eigene Musikkapelle, deren Leiter der Zinkenist Pietro Verdina war ${ }^{9}$. Ferdinands jüngerer Bruder Leopold Wilhelm, inzwischen Bischof von Passau, Straßburg und Olmütz, Hoch- und Deutschmeister des Deutschen Ordens und Oberbefehlshaber der kaiserlichen Truppen, dachte erst 1640/41 daran, sich auch eine solche Institution zu leisten. Der kaiserliche Hofmusiker Lodovico Bartolaia schlug ihm in einem Brief vom März 1641 den Fagottisten Antonio Bartoli als Vize- oder Kapellmeister vor ${ }^{10}$. Schon $1640^{11}$ und nochmals 1641 beauftragte der Erzherzog den Komponisten Giacinto Cornacchioli, ihm in Italien, vor allem aus Rom, eine Reihe guter Musiker zu verpflichten und Instrumente sowie Musikalien anzukaufen. Dessen Briefe an den Auftraggeber und an dessen Rat Hermann Graf von Attems aus den Jahren 1641 und $1642^{12}$ sind eine wichtige Quelle für diese Bemühungen. Für den Kapellmeisterposten wurden in Rom die Berühmtheiten Giacomo Carissimi und Francesco Foggia vergeblich angefragt; der Erzherzog betraute schließlich den genannten Pietro Verdina mit diesem Amt, obwohl dieser inzwischen schon kaiserlicher Vizekapellmeister war. Er musste mit dem Generalissimus ins Feld ziehen und geriet im November 1642 bei Breitenfeld mit anderen Musikern vorübergehend in schwedische Kriegsgefangenschaft; im darauffolgenden Juli 1643 verstarb er.

Zusammen mit dem Briefwechsel der Brüder seit $1627^{13}$ bilden die in Stockholm überlieferten und demnächst zu publizierenden ${ }^{14}$ Briefe Leopold Wilhelms aus den Jahren 1646 und 1647 an den Kaiser eine sehr persönliche Quelle für beider dichterische und kompositorische Tätigkeiten und auch für ihre Kapellen. Denn der Erzherzog schreibt am 8. November 1647 aus Gent an seinen Bruder, er bekomme „gewaltige recruten in Musica“, die er auch gleich nennt, und etwa drei Wochen danach aus Brüssel, wo er nun als Statthalter der Spanischen Niederlande residierte, wieder: „Mein Music wachst gewaltig, galing fircht Ich auf einmal geet Sie zue grunt; auß Mangl der Mitl, wan un gott nit pald den fried schickt." Das beweist, dass er nach der ersten Aufnahmephase 1642 gegen

8 SEIFERT, Herbert. Kirchers Kompositionsschüler an den Habsburgerhöfen: Organisten und Kaiser In Melanie Wald-Fuhrmann (Hg.). Steinbruch oder Wissensgebäude? Zur Rezeption von Athanasius Kirchers Musurgia universalis in Musiktheorie und Kompositionspraxis (Bibliotheca Helvetica Romana XXXIV). Basel: Schwabe, 2013, S. 131-141.

9 Zu diesem s. SEIFERT, Herbert. Giovanni Sansoni, Cornettist, Maestro dei concerti and Composer in Venice, Graz and Vienna In De musica disserenda XIII/1-2, 2017, S. 147-156. (URL: https://ojs.zrc-sazu.si/dmd/ article/view/6582/6234).

10 SAUNDERS, Steven. Sacred Music at the Hapsburg Court of Ferdinand II (1615-1637): The Latin Vocal Works of Giovanni Priuli and Giovanni Valentini. Diss. University of Pittsburgh 1990/Ann Arbor: University Microfilms International, S. 903.

11 Siehe den Beitrag von Marko DEISINGER in diesem Band.

12 ANTONICEK, Theophil. „Pigliar musici dall'Italia”. Ein Agent des Erzherzogs Leopold Wilhelm auf der Suche nach italienischen Musikern: Giacinto Cornacchioli In Studien zur Musikwissenschaft 9, 2017, S. 7-60.

13 Wien, Haus-, Hof- und Staatsarchiv, Habsburgisch-lothringisches Familienarchiv (Hausarchiv), Familienarchiv A, Karton 11.

14 Von einer Arbeitsgruppe an der Universität München unter der Leitung von Prof. Mark Hengerer. 
Ende 1647, bald nach seiner Ankunft in Brüssel, eine Vergrößerung seiner Hofmusik durchführte, da er nun nach dem unsteten Leben als Feldherr mehr Gelegenheiten für Unterhaltungen und Repräsentationen hatte. Erst dadurch war es ihm schließlich auch möglich, 1650 die erste Oper in Brüssel aufführen zu lassen, Ulisse nell'Isola di Circe mit Musik seines neuen Kapellmeisters Gioseffo Zamponi.

Schon im Februar 1646 schreibt der Kaiser an seinen Bruder, dass ihm dessen übersandtes Drama sehr gefalle und er das Urteil seines Hofkapellmeisters Giovanni Valentini, der auch Dichter dramatischer Texte war, hören wolle „und ob ers Componiren will“"15. Es ist durchaus wahrscheinlich, dass dies das Libretto zu der von Ferdinand später selbst komponierten, schon genannten Oper war, die unter der lateinischen Gattungsbezeichnung Drama musicum in der Literatur aufscheint und deren seit ihrer Nennung im Revisionsbericht Guido Adlers zu seiner Edition von Teilen daraus ${ }^{16}$ verschollen gewesene zeitgenössischen Handschrift ich aufgespürt habe

\section{Exkurs 1: Ferdinands III. Drama musicum}

„Drama musicum compositum ab Augustissimo Ferdinando III Pio ... et ab eodem ad P. Athanasium Kircherum Transmissum anno 1649“ (Abb. 1) lautet der Titel dieser Partitur, die Guido Adler für das wahrscheinliche Dedikationsexemplar des Kaisers an Kircher hielt. Sie befand sich damals nach Adlers Aussage im Besitz von Alexander Posonyi. Dieser war Kunst- und Autographensammler und -händler in Wien, wie mehrere Auktionskataloge belegen. Nach seinem Tod wurde seine Sammlung vom Antiquariat Friedrich Cohen in Bonn angeboten. Die im 2. Teil dieses Auktionskatalogs ${ }^{17}$ angebotene Partitur erhält dort in französischer Sprache eine Beschreibung, die vor allem durch ihre Umfangsangabe zu meiner Identifizierung geführt hat.

In Wortlaut und Zeileneinteilung identisch mit dem Originaltitel sind die Aufschriften auf zwei Kopien der Oper aus der ersten Hälfte des 19. Jahrhunderts, eine in der Bayerischen Staatsbibliothek in München ${ }^{18}$, die von Johann Caspar Aiblinger 1833 in Rom wohl um den auf der Handschrift vermerkten Preis von 2 scudi 70 gekauft worden war $^{19}$, und eine in der Musiksammlung der Österreichischen Nationalbibliothek in Wien ${ }^{20}$, die bald danach durch den Schreiber der kaiserlichen Hofbibliothek Anton

15 ANTONICEK, Theophil. Musik und italienische Poesie, S. 1.

16 ADLER, Guido (Hrsg.). Musikalische Werke der Kaiser Ferdinand III., Leopold I. und Joseph I. Bd. 2. Wien: Artaria 1892, S. 305.

17 COHEN, Friedrich. Autographen-Sammlung Alexander Posonyi in Wien. II. Musiker. Meist kostbare Briefe und Musikmanuskripte der berühmtesten Komponisten, Virtuosen und Musikschriftsteller aller Zeiten und Völker nebst einer kleinen Auswahl von Musikdrucken u. Werken zur Musik-Literatur (Friedrich Cohen, Catalog 98). Bonn: Carl Georgi 1900, S. 29.

18 D-Mbs Coll.Mus.Max.99. Nähere Beschreibung bei WACKERNAGEL, Bettina. Bayerische Staatsbibliothek. Katalog der Musikhandschriften 3. Collectio musicalis Maximilianea. München: Henle 1981, S. 37.

19 AmZ 1833, Sp. 854.

20 A-Wn Mus.Hs.16014. 


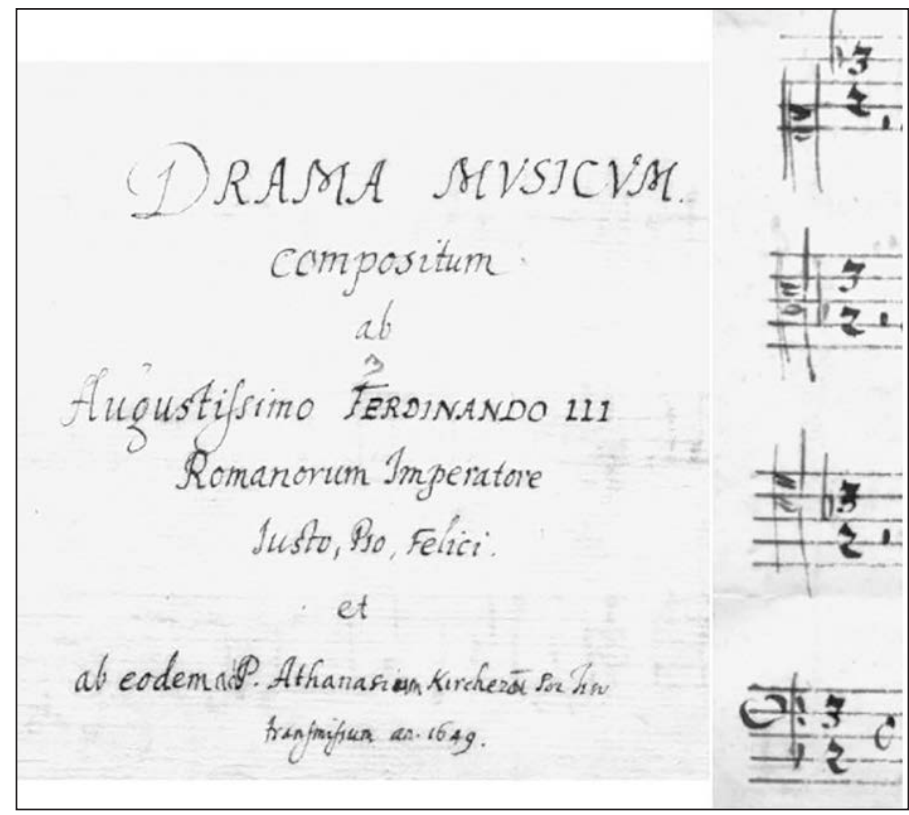

Abb. 1 Titelblatt und Schriftmuster zu Ferdinands III. Drama musicum, loco secreto

Schmid $^{21}$ von dieser Münchner Abschrift kopiert worden ist ${ }^{22}$, was Adler nicht bekannt war, denn er vermutete als Datierung der Abschrift Ende des 18. oder Anfang des 19. Jahrhunderts ${ }^{23}$. Nach den von Adler - nach der Wiener Kopie, jedoch unter teilweiser Berücksichtigung der alten Handschrift - edierten Auszügen ${ }^{24}$ ist 2015 die erste komplette Edition erschienen ${ }^{25}$, allerdings ausschließlich nach der Wiener Quelle (Anton Schmids Kopie) ohne die auf die Originalquelle zurückgehenden Korrekturen Adlers; die übrigen Quellen waren den Herausgebern nicht zugänglich oder - die wiederentdeckte - nicht bekannt. Diese fehlerhafte Edition diente mehreren Aufführungen seit 2014 als Grundlage. Ich plane eine Neuedition dieses ersten erhaltenen Wiener Musikdramas nach der zeitgenössischen Quelle.

21 Den Schreiber identifiziert ANTONICEK. Musik und italienische Poesie, loc. cit. Der OPAC der Österreichischen Nationalbibliothek nennt kurioser Weise Athanasius Kircher als Schreiber!

22 Siehe SCHLAGER, Johann Evangelist. Über das alte Wiener Hoftheater In Sitzungsberichte der kaiserlichen Akademie der Wissenschaften. Philosophisch-historische Classe. 6. Bd. Wien: 1851, S. 147-171: 161.

23 ADLER. loc. cit.

24 ADLER. S. 5-26. Offenbar ein Helfer des Herausgebers hat die durch „Dr. Janeczek“ (so die Unterschrift auf der letzten Seite der im Archiv der Denkmäler der Tonkunst in Österreich unter der Signatur „Kaiserwerke A 74“ erhaltenen Kopie mit der Datierung III/1891) von der Wiener Kopie angefertigte Abschrift für die zu publizierenden Teile nach dem Originalmanuskript (damals im Besitz von Alexander Posonyi) korrigiert, u.a. zahlreiche Taktstriche getilgt und die rhythmisch gravierend falsche Transkription des Colors richtiggestellt.

25 HOFSTÖTTER, Rudolf und RAINER, Ingomar (Hrsg.). Kaiser Ferdinand III. Drama musicum (Wiener Edition alter Musik 43). Wien: Doblinger 2015. 
Die Musikwissenschaftlerin Erika Honisch hat neuerdings einen eindeutigen Beleg für eine Aufführung dieser Oper am Kaiserhof in Prag schon im Februar 1648 gefunden, der auch Leopold Wilhelm als Autor des Librettos bestätigt. Details über diese Funde werden in gesonderten Publikationen gegeben werden.

Danach werden in Briefen des Erzherzogs immer wieder Sonette, Madrigale und andere Dichtungsformen seiner Erfindung genannt, die er über die Texte des kaiserlichen Kapellmeisters Giovanni Valentini stellt, und in den Gegenbriefen Kompositionen des Kaisers, die dieser ebenfalls vorteilhaft mit solchen Valentinis vergleicht. Schon 1640 hatte Ferdinand an seinen Bruder geschrieben: „Ich hab auch dise Fasten ein Miserere gemacht [...]. Ich Main es khin mit allen des Valentin miserere competirn, sunderlich in ripienis ist Vniversaliter gelobt worden." ${ }^{\prime 6}$

\section{Exkurs 2: Giovanni Priulis Flucht}

Zu diesem Giovanni Valentini und seinem Vorgänger im Kapellmeisteramt sei ein Exkurs mit neuen Forschungsergebnissen gestattet: Es war bisher rätselhaft, weshalb dieser Organist Kaiser Ferdinands II. noch zu Lebzeiten des bisherigen Hofkapellmeisters Giovanni Priuli, am 15. Juni $1626^{27}$, zu seinem Nachfolger bestellt wurde; Priuli schrieb sein Testament auf der Festung Klamm bei Schottwien, die auf dem Weg von Wien nach Italien liegt, am 18. Juli eigenhändig und ohne Zeugenunterschriften und verstarb kurz danach, was sich daraus ergibt, dass es am 26. Juli publiziert wurde ${ }^{28}$. Der Grund seiner Abreise aus Wien und seiner Entlassung aus dem kaiserlichen Dienst erhellt aus einem Brief der Fürstin Polyxena Pernstein-Lobkowitz an ihren Mann Zdenko Adalbert Popel Lobkowitz vom 23. Mai 1626. Sie schreibt aus Prag: „Mi rey, tanbien se dise que un musico del Amo muy faboresido es partido o hoydo por lo mismo del Berca y que a escrito al Amo que lo que hasia que era por ser tan caloroso que si lo hisiera con mujeres, que costava la vista. Que por esto para conservalla uvo de tomar este remedio. iNunca e oydo era Bueno para la vista! Y que dira que ay más que un par de dosenas de gente onrada y grandes que hasen lo mismo." ${ }^{29}$ Also: Man sage, dass ein vom Kaiser sehr geschätzter Musiker abgereist bzw. geflohen sei,

26 Wien, Haus-, Hof- und Staatsarchiv, Habsburgische Familienkorrespondenz A, Karton 11, Fasc. 2, fol. 40v. Die Lesart von SAUNDERS. Sacred Music, S. 884, weicht davon ab. S. auch ANTONICEK. Musik und italienische Poesie, S. 4.

27 KNAUS, Herwig. Die Musiker im Archivbestand des kaiserlichen Obersthofmeisteramtes (1637-1705) (Veröffentlichungen der Kommission für Musikforschung der Österreichischen Akademie der Wissenschaften 8), Band 2. Wien: Verlag der Österreichischen Akademie der Wissenschaften, 1968, S. 132.

28 SAUNDERS. Sacred Music, S. 340f., 878.

29 MAREK, Pavel (ed). Svědectví o ztrátě starého světa. Manželská korespondence Zdeňka Vojtěcha Popela z Lobkovic a Polyxeny Lobkovické z Pernštejna. České Budějovice: Historický Ústav Jihočeské Univ. 2005, S. 465-467. Vladimír Maňas danke ich dafür, dass er mich auf diese Briefstelle aufmerksam gemacht hat, Andrea SommerMathis und Christopher Laferl für sprachliche Hilfe beim Verständnis der etwas kryptischen spanischen Formulierungen. 
aus demselben Grund wie Berka (d. i. Jan Theodor Berka von Dubá und Lipá, der im Jahre 1625 der Vergewaltigung einiger seiner weiblichen Untergebenen schuldig erklärt worden war). Der Musiker habe seinem Herrn geschrieben, dass er das getan habe, weil er so heißblütig sei; wenn er es mit Frauen gemacht hätte, so hätte es ihn seine Sehkraft gekostet. Um diese zu behalten, hätte er zu diesem Mittel greifen müssen - eine sehr befremdliche, auch von der Fürstin angezweifelte Rechtfertigung.

Wegen der zeitlichen Koinzidenz ist mit diesem Musiker mit höchster Wahrscheinlichkeit der unverheiratete Hofkapellmeister Giovanni Priuli gemeint, und möglicherweise war der Kündigungsgrund, dass er sich sexuell an Kapellknaben vergangen hat, die wohl seiner Obhut anvertraut waren und jedenfalls seine Untergebenen waren. Nach seiner Flucht, die jedenfalls vor Mitte Mai stattgefunden haben muss, wurde also sehr schnell, am 15. Juni, der bisherige Organist der Hofkapelle Valentini zu seinem Nachfolger ernannt. Am 25. Juni nahm eine Hofkommission ein „Inventarium aller und jeder Sachen, so sich in Ihrer Kay[serlichen] May[estät] gewesten Capelmaisters Johann Priuli Zimmer befunden“, auf, darunter Kleidung, Möbel, Silber, Musikinstrumente, Bücher und Musikalien $^{30}$. Priuli hatte also bei seiner Flucht aus Wien offenbar den Großteil seiner beweglichen Güter zurückgelassen. Die Musikalien vermachte er in seinem Testament dem Kaiser, das Silber dem Botschafter der Republik Venedig am Kaiserhof sowie Instrumente und Goldmedaillen Freunden in der Hofmusikkapelle ${ }^{31}$. Seine Todesursache ist nicht bekannt.

Es ist aufschlussreich, zu verfolgen, wie sich das Verhältnis der Habsburger-Brüder zu Valentini, dem Hofkapellmeister schon ihres Vaters und auch gemeinsamen Lehrer in Musik und Poesie ${ }^{32}$, im Lauf der Jahre ändert. Ist es in ihrer Jugend Bewunderung, wie sie sich etwa in einem Brief des 20jährigen Leopold Wilhelm von 1634 ausdrückt: „Der

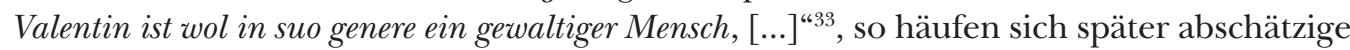
Äußerungen über seine Dichtungen und Kompositionen. Der Erzherzog schreibt am 4. Februar 1646 aus dem Feld über die damals gerade erschienenen Rime sacre Valentinis ${ }^{34}$ : „ist mir nur la[i]d, daß e $\beta$ geistlich ist, daß man e $\beta$ nit also au $\beta$ lachen khan, wie Sie es meritieren; [...] gleich alß Ich es gelesen habe, [habe ich] diese zwo risposten darauff gemacht [...]; aber wan es $E$ [ure] $K$ [aiserliche] $M$ [ajestät] und die kayserin gelesen haben, so verprenen e $\beta E K M$; dan Ich nit wolt daß e $\beta$ der Valentini erfaren that, e $\beta$ that dem gueten alten schmerzen; ..."35. Trotzdem haben ihn die zahlreichen Anagramme in dieser Sammlung dazu angeregt, sich selbst in dieser ars combinatoria zu betätigen; einen Monat danach schreibt er nämlich: „der anagramitsche spiritus deß Valentini hatt mir die anagramatische uena erefnet wie

30 SAUNDERS. Sacred Music, S. 340f., 878.

31 Ibid. S. 341f.

32 SCHREIBER. S. 30.

33 Ibid. S. 47, Anm. 23. Zitat aus einem Brief des Erzherzogs an seinen Bruder vom 17. Juli 1634.

34 Linz: Maria Kürner 1646.

35 Stockholm, Riksarkiv Extranea 195, XVI. Tyskland, a. Handlingar och brev, 3. Archivfragment Kejsar Ferdinand III:s arkiv 1646-1648. Brief des Erzherzogs Leopold Wilhelm an Kaiser Ferdinand III. vom 4. Februar 1646. 
Die Brüder Ferdinand III. und Leopold Wilhelm, ihre Stellung zu ihrem Lehrer Giovanni Valentini, ...

EKM hie bei sehen; mit nachsten khumbt etwas gar schen $\beta,[. . .]^{\text {“36, }}$ und damit meint er wohl das Sonett, das er dem Kaiser fünf Tage später sendet und „welch $\beta$ Ich main daß peste ist, daß Ich mein lebtag gemacht habe; [...] wan Ich mein iudicium selbst sagen soll, so ware e $\beta$ mir lieber al $\beta$ de $\beta$ Valentini sein ganz piechl; will gern Ihr M[ajestät] und de $\beta$ Valentini Judicium dariber vernemen. "37

Im November 1647 schreibt er aus Brüssel gar: „gestern habe ich von dem Valentin poesien empfangen, e $\beta$ seint aber nicht rechte poesien, sonder retro grada, [...], wan [er] E $\beta$ nuhr nit trukhen lie $\beta$, und in musica sezt, welch $\beta$ doch gar nit woll lauten mu $\beta$, Ich scham mich recht, wan solche sachen unter die leid khumen;“38. Man könnte das auf den ersten Blick für Überheblichkeit des adeligen Dilettanten gegenüber dem professionellen Poeten und Lehrer halten, doch eine Überprüfung zeigt, dass der genannte Druck Valentinis von 1646 tatsächlich viele Palindrome, Anagramme und sonstige Wort- und Buchstaben-Spielereien wie Binnenreime, bis zu zwölf reimidente Verse oder z. B. Verse, die alle mit bzw. ohne den Buchstaben R gedichtet sind, enthält und kaum wirkliche Poesie (Abb. 2-3). Insofern kann ich diese Kritik Leopold Wilhelms sehr gut nachvollziehen, und eine

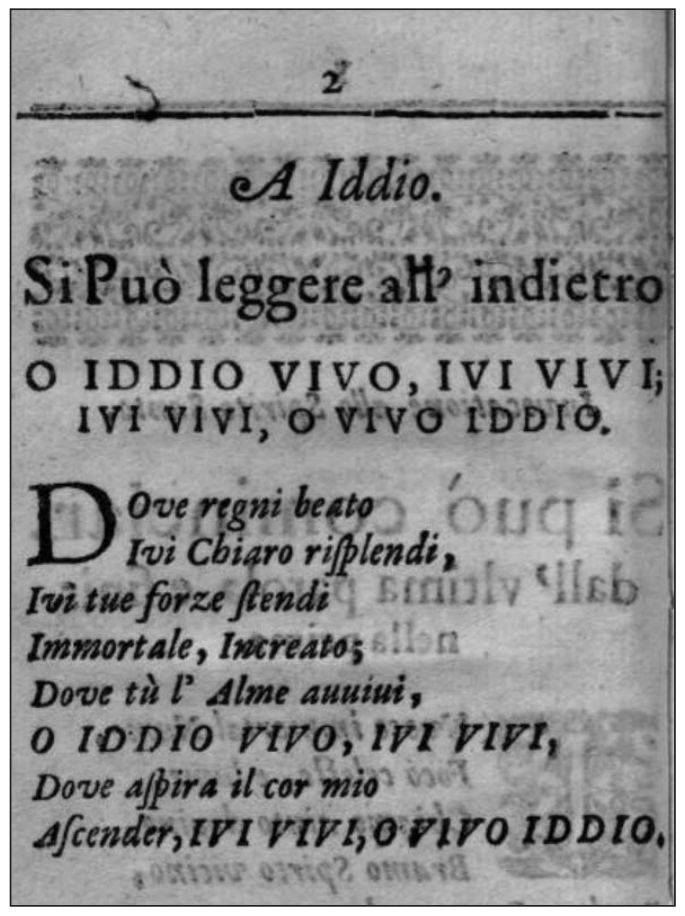

Abb. 2 Giovanni VALENTINI. Rime sacre, S. 2 (Palindrom)

\begin{tabular}{|c|c|c|c|}
\hline \multicolumn{4}{|c|}{216} \\
\hline \multicolumn{4}{|c|}{$\begin{array}{l}\text { Peccatore, che defid } \\
\text { prima di morir far penitenza. }\end{array}$} \\
\hline \multicolumn{4}{|c|}{ Ogni parola, è in Anagramma. } \\
\hline$M O R T E$ & $S A N$ & $\triangle N G V E$ & $L E G G E$ \\
\hline MERTO, & $S T I A N$ & $\forall A D I$ & $N O V A$ \\
\hline$V E R G A$ & $M E C O$ & $V E N G A$ & $L \mathcal{L} G G \varepsilon$, \\
\hline$G R A V E$ & $G O M E$ & $D I V A$ & $V A N O$ \\
\hline $\boldsymbol{L} A S S O$ & $B R A M O$ & $S A N T A$ & $T R E M A$ \\
\hline$I^{\prime} O S S A$ & $O M B R A$ & $S A T A N$ & MARTE. \\
\hline$M^{\prime} A P R I$ & $R I A$, & $C A D I$ & $C E R C O$ \\
\hline$P R I M A$ & $I R A$ & CONTRO & CROCE, \\
\hline$O D I$ & $D A N N O$ & TRONCO & POI \\
\hline DIO & STR ANO & MORA; & PIO \\
\hline$M I A$ & NOSTRA & $D I C A$ & VEDO \\
\hline$I M A$ & $D O N N A$ & $S E R P E$ & DOVE \\
\hline$A L M A$ & $L E V I$ & PER SE; & MORTE \\
\hline$M A L A ;$ & $P I L E$ & $|\mathrm{ROMA}|$ & MERTO \\
\hline
\end{tabular}

Abb. 3 Giovanni VALENTINI. Rime sacre, S. 216 (Anagramme)

36 Ebenda, Brief vom 7. März 1646.

37 Ebenda, Brief vom 12. März 1646.

38 Ebenda, Brief vom 23. November 1647. 
literaturwissenschaftliche Untersuchung der Entwicklung des Dichters Valentini könnte da wohl Aufschluss bieten; in diesen letzten Lebensjahren bis zu seinem Tod 1649 hat er tatsächlich fast nur mehr Anagramme veröffentlicht: 120 über den Heiligen Xaver (Saverio) $1646^{39}$ und 134 über die Heilige Katharina im Jahr $1647^{40}$, und 1649 noch eine Sammlung über den Namen Jesus ${ }^{41}$.

Hier muss daran erinnert werden, dass er nicht Geistlicher war, sondern zweimal verheiratet und Vater mehrerer Kinder. Trotzdem hat sich Valentini in seinen Dichtungen, die er seit 1642 veröffentlicht hat, fast ausschließlich mit geistlicher Thematik befasst und scheint auch streng auf die Einhaltung der kirchlichen Gesetze geachtet zu haben, denn Leopold Wilhelm schreibt im März 1646 aus dem Feldlager: „[...] habe [...] ein [...] soneto gemacht; $[. .$.$] daß sich der Valentini nitt scandaliziere, da \beta$ ich in der fasten etwa $\beta$ verlieb $\beta$ mache; und daß Ich wider in mein alten gueten concept khume, so schick Ich diese geistliche composition, $[\ldots]^{\prime \prime}$, die er durch drei seiner Hofmusiker komponieren lassen wolle ${ }^{42}$. Es ist möglich, dass er diesen Text zehn Jahre später in seiner Sammlung Diporti del Crescente als Oratorio Per la Settimana santa in Musica veröffentlicht hat ${ }^{43}$.

Dass Kaiser Ferdinand seine eigenen Kompositionen aber auf eine Stufe mit den wirklich meisterlichen seines Kapellmeisters stellt, scheint mir in Kenntnis einiger davon dagegen nicht berechtigt zu sein. Als kurzes Beispiel dafür soll nur der Schluss des „Miserere“ Ferdinands III. für 12 Singstimmen dienen, das er mit Valentinis Komposition vergleicht und das - wie zitiert - besonders in den Tutti-Passagen sehr gelobt worden sei. Wir sehen hier die für seine Musik typischen vielfachen Wiederholungen desselben Motivs auf einer oder verschiedenen Tonstufen.

Abgesehen von liturgischen Kompositionen hat Ferdinand III. immer wieder italienische Dichtungen seines Bruders vertont, wie dieser auch im Vorwort seiner Sammlung angibt $^{44}$. Insgesamt sind 20 Kompositionen des Kaisers erhalten und mindestens weitere 43 dokumentiert; von diesen 63 ist die Überzahl geistlich mit lateinischen Texten; nur 12 sind italienisch, davon vier nachweislich zu Texten von Leopold Wilhelm - eines davon ein frühes Weihnachtsoratorium ${ }^{45}$ - und zwei mit eigenen Texten von Ferdinand.

39 Cento e venti Anagrammi Sovra Santo Saverio Apostolo dell'Indie, Wien: Matthaeus Cosmerovius 1646.

40 Cento e trenta quattro anagrammi sovra il glorioso nome di Santa Caterina martire, con nove ottave obligate, Wien: Matthaeus Cosmerovius 1647

41 Anagrammata supra nomen Jesum, Jesu, Jesus ante sanctam communionem, Wien: Matthaeus Cosmerovius 1649 .

42 Stockholm, loc. cit., Brief vom 10. März 1646.

43 In der Österreichischen Nationalbibliothek gibt es auch eine handschriftliche Version (Cod. Ser. nov. 4270), die mit 4. April 1654 datiert ist. S. dazu SEIFERT, Herbert. The Beginnings of Sacred Dramatic Musical Works at the Imperial Court of Vienna: Sacred and Moral Opera, Oratorio and Sepolcro In BESUTTI, Paola (ed.). L'Oratorio musicale italiano e $i$ suoi contesti (secc. XVII-XVIII) (Quaderni della Rivista italiana di musicologia 35), Firenze: Olschki 2002, S. 489-511: 499-500; auch in id. Texte zur Musikdramatik im 17. und 18. Jahrhundert. Aufsätze und Vorträge. Hrsg. v. Matthias J. Pernerstorfer (Summa Summarum 2), Wien: Hollitzer 2014 , S. 765-782: 771 . 
Die Brüder Ferdinand III. und Leopold Wilhelm, ihre Stellung zu ihrem Lehrer Giovanni Valentini, ...
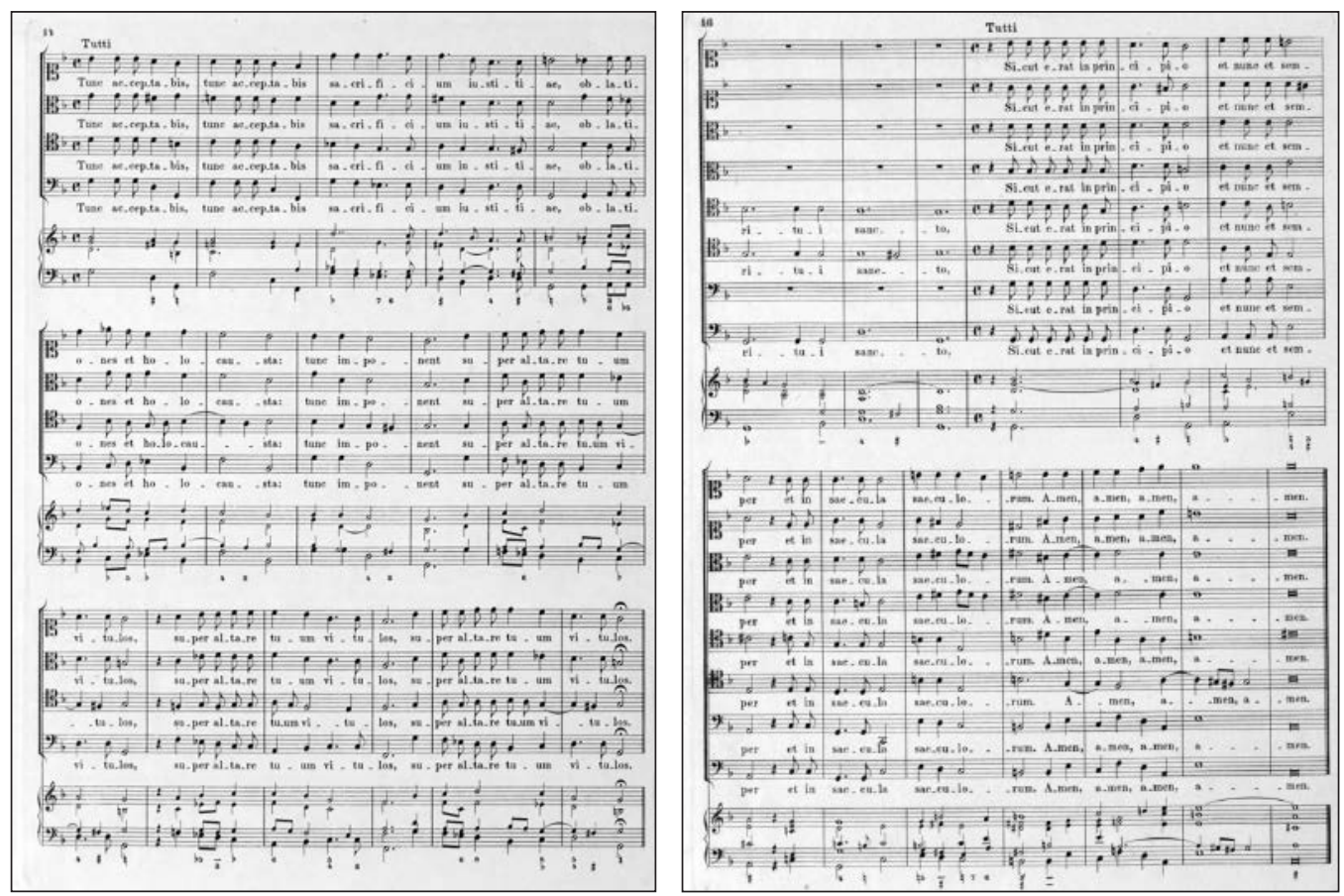

Abb. 4-5 Ferdinand III., Miserere, Schluss (aus ADLER. Bd. 1, S. 14 und 16)

Eine durch die vor kurzem publizierten und die zur Publikation vorbereiteten einschlägigen Briefe mögliche Übersicht über die vor allem italienischen Kammermusiker, Poeten und Architekten des Kaiserbruders Erzherzog Leopold Wilhelm aus den Jahren 1640 bis zu seinem Tod 1662 soll abschließend geboten werden. In diesen gut zwei Jahrzehnten waren etwa 32 namentlich bekannte Personen in seiner „Musica di camera“, nicht inkludiert die Musiker der geistlichen Hofkapelle von Brüssel, die auch vor und nach Leopold Wilhelms Statthalterschaft dort wirkten. 
Die Brüder Ferdinand III. und Leopold Wilhelm, ihre Stellung zu ihrem Lehrer Giovanni Valentini, ...

\section{Musica di Camera Leopold Wilhelms}

Kapellmeister

\begin{tabular}{|l|l|l|l|}
\multicolumn{1}{c}{ Name } & \multicolumn{1}{c}{ Lebensdaten } & Anstellung & \multicolumn{1}{c|}{ ?-Juli 1643 } \\
\hline Pietro Verdina & c1600-1643 & Nov.1644-Jan. 1646 & \\
\hline Orazio Benevoli & $1605-1672$ & mind.1646-1648 & dann Vize \\
\hline $\begin{array}{l}\text { Giovanni Pietro Finatti, } \\
\text { auch T. }\end{array}$ & & 1648-mind. 1657 & $\begin{array}{l}\text { dann HK des Kurfürsten. } \\
\text { v. Köln }\end{array}$ \\
\hline Gioseffo Zamponi & $1610 / 20-1662$ & & \\
\hline
\end{tabular}

Vizekapellmeister

\begin{tabular}{|l|l|l|l|}
\hline $\begin{array}{l}\text { Giovanni Pietro Finatti, } \\
\text { auch T, Komp. }\end{array}$ & $1648-1652$ & dann Neuburg \\
\hline
\end{tabular}

Sänger

\begin{tabular}{|c|c|c|c|}
\hline Giorgio Ostresio, S & c1621-1669 & 1641?-1657? & dann kais. HK \\
\hline $\begin{array}{l}\text { Giovanni Battista } \\
\text { Riccioni, S }\end{array}$ & & 1642-mind.1656 & \\
\hline $\begin{array}{l}\text { Hans Caspar Richter, S, } \\
\text { A, Org. }\end{array}$ & & $\begin{array}{l}\text { mind.1642-1646 S, } \\
\text { 1647-mind.1652 A } \\
\text { u./o. Kopist o. Organist. }\end{array}$ & \\
\hline $\begin{array}{l}\text { Giovanni Battista } \\
\text { Mocchi, S, Komp., } \\
\text { auch Org. }\end{array}$ & $1620-1688$ & Nov. $1647-1650$ & $\begin{array}{l}\text { dann Kapellmeister in } \\
\text { Neuburg }\end{array}$ \\
\hline $\begin{array}{l}\text { Giovanni Francesco } \\
\text { Castri, A oder T }\end{array}$ & & ?-vor Juni 1648 & \\
\hline $\begin{array}{l}\text { Antimo Liberati, A, } \\
\text { Komponist }\end{array}$ & $1617-1692$ & c1640-1642 & dann in Rom \\
\hline $\begin{array}{l}\text { Venanzio Leopardi, A } \\
\text { oder T }\end{array}$ & $1617-1692$ & $\begin{array}{l}\text { mind.seit Aug.1641- } \\
1643 \text { ? }\end{array}$ & $\begin{array}{l}\text { seit } 1643 \text { ? bei Eleonora, } \\
\text { dann Bologna, Rom }\end{array}$ \\
\hline Steffano Boni, T & ?-1688 & Nov.1647-1652 & $\begin{array}{l}\text { dann Dresden, ab } 1654 \\
\text { kais. HK }\end{array}$ \\
\hline $\begin{array}{l}\text { Francesco Maria } \\
\text { Riccioni, T, Kaplan }\end{array}$ & & 1642-mind. 1645 & \\
\hline $\begin{array}{l}\text { Antonio Abati, B, auch } \\
\text { Poet }\end{array}$ & $1603-1667$ & 1642?-mind.1651 & dann Brüssel, Italien \\
\hline Gabriele Ansalone, B & & $\begin{array}{l}1642 ? \\
\text { u.mind.1644-1652 }\end{array}$ & $\begin{array}{l}\text { dann München, } \\
\text { Neuburg }\end{array}$ \\
\hline $\begin{array}{l}\text { Francesco Incarnatini, } \\
\text { B, Komp. }\end{array}$ & & März 1642-1645? & Bildhauer, Maler \\
\hline Benedetto Andreasi & & mind. 1641-1642 & $\begin{array}{l}\text { auch Rom, dann bei } \\
\text { Eleonora I. }\end{array}$ \\
\hline $\begin{array}{l}\text { Giacinto Cornacchioli, } \\
\text { auch Agent, Organist }\end{array}$ & 1598/99-nach 1673 & mind.Feb.1640-1642 & $\begin{array}{l}\text { dann Rom, Fermo, } \\
\text { Ascoli }\end{array}$ \\
\hline
\end{tabular}


Die Brüder Ferdinand III. und Leopold Wilhelm, ihre Stellung zu ihrem Lehrer Giovanni Valentini, ...

\section{Instrumentalisten}

\begin{tabular}{|l|l|l|l|}
\hline Roberto Sabbatini, Vl. & ?-1692/93 & Nov.1647-1650 & $\begin{array}{l}\text { dann kais.HK, München, } \\
\text { Innsbruck,Neuburg }\end{array}$ \\
\hline $\begin{array}{l}\text { Alessandro Subissati, } \\
\text { Vl., Komp. }\end{array}$ & $?-1677$ & mind. 1644 & seit c1645 Warschau \\
\hline $\begin{array}{l}\text { Domenico Serragli de } \\
\text { Conti, VI?,Kaplan,Komp. }\end{array}$ & c1612-1688 & mind.1647-mind.1652 & \\
\hline $\begin{array}{l}\text { Paul Pickhl } \\
\text { von Pickhlfeld, VdG }\end{array}$ & mind.1647-1650 & dann kais.HK \\
\hline $\begin{array}{l}\text { Bartholomäus Albrecht, } \\
\text { Tr. }\end{array}$ & c1614-1654 & mind.1643-1648 & dann kais.Dienste \\
\hline $\begin{array}{l}\text { Andreas Wagenhueber, } \\
\text { mus.Tr. }\end{array}$ & $c 1634-1701$ & c1656-? & dann kais.Dienste \\
\hline Jacob Schmidt, Tr. & c1629-1679 & mind.1660-1662 & dann Olmütz? \\
\hline $\begin{array}{l}\text { Johann Friedrich Hel- } \\
\text { wig, Pos. }\end{array}$ & p-16687-1650 & dann kais.HK \\
\hline $\begin{array}{l}\text { Christoph Magnus } \\
\text { Neumann, Pos. }\end{array}$ & $1627-1693$ & $1647-$ mind.1653 & dann kais.HK \\
\hline $\begin{array}{l}\text { Johann Caspar Kerll, } \\
\text { Org., Komponist }\end{array}$ & $1627-1701$ & $1648-1652$ & dann München, Wien \\
\hline $\begin{array}{l}\text { Abraham van } \\
\text { den Kerckhoven, } \\
\text { Org.,Komp. }\end{array}$ & $1612 / 1620-1672$ & Feb.1647-mind.1648 & danach Rom \\
\hline $\begin{array}{l}\text { Antonio Francesco } \\
\text { Tenaglia, Cemb., } \\
\text { Lt.,Komp. }\end{array}$ & vor 1647-1655 & \\
\hline
\end{tabular}

Unbestimmbare Musiker

\begin{tabular}{|l|l|l|l|}
\hline Carlo Carmignano & mind.1644 & \\
\hline Ludovico Porta & & mind.1647-mind.1655 & \\
\hline
\end{tabular}

\section{Kopist}

\begin{tabular}{|l|l|l|l|}
\hline Johann Weldschütz & c1601-1674 & mind.1644-1650 & dann kais.HK \\
\hline
\end{tabular}

Poeten

\begin{tabular}{|l|l|l|l|}
\hline Orazio Persiani & & 1642 & davor Venedig \\
\hline Antonio Abati, auch B & c1603-1667 & 1642 ?-mind.1651 & dann Brüssel, Italien \\
\hline
\end{tabular}

Bühnenarchitekt

\begin{tabular}{|l|l|l|l|}
\hline Giovanni Battista Angelini & ?-Jan.1659 & 1648-1657 & dann Kaiserhof \\
\hline
\end{tabular}

\section{Ballettmeister}

\begin{tabular}{|l|l|l|l|}
\hline Giovanni Battista Balbi & & Feb.1648-1650 & danach Venedig, Paris \\
\hline Santo Ventura & vor 1600-1676 & $\begin{array}{l}\text { mind.Aug.1648-mind. } \\
\text { Juni 1649 }\end{array}$ & $\begin{array}{l}\text { davor u.danach } \\
\text { Kaiserhof }\end{array}$ \\
\hline
\end{tabular}




\section{Bibliography}

\section{Sources}

Stockholm, Riksarkiv Extranea 195, XVI. Tyskland, a. Handlingar och brev, 3. Archivfragment Kejsar Ferdinand III:s arkiv 1646-1648.

Wien, Haus-, Hof- und Staatsarchiv, Habsburgische Familienkorrespondenz A, Karton 11.

\section{Literature}

ADLER, Guido (Hrsg.). Musikalische Werke der Kaiser Ferdinand III., Leopold I. und Joseph I. Bd. 2. Wien: Artaria 1892.

Allgemeine musikalische Zeitung 1833, Sp. 854.

ANTONICEK, Theophil. „Pigliar musici dall'Italia”. Ein Agent des Erzherzogs Leopold Wilhelm auf der Suche nach italienischen Musikern: Giacinto Cornacchioli In Studien zur Musikwissenschaft 9, 2017, S. 7-60.

ANTONICEK, Theophil. Musik und italienische Poesie am Hofe Kaiser Ferdinands III. (Mitteilungen der Kommission für Musikforschung 42). Wien: Verlag der Österreichischen Akademie der Wissenschaften, 1990.

COHEN, Friedrich. Autographen-Sammlung Alexander Posonyi in Wien. II. Musiker. Meist kostbare Briefe und Musikmanuskripte der berühmtesten Komponisten, Virtuosen und Musikschriftsteller aller Zeiten und Völker nebst einer kleinen Auswahl von Musikdrucken u. Werken zur Musik-Literatur (Friedrich Cohen, Catalog 98). Bonn: Carl Georgi 1900.

HOFSTÖTTER, Rudolf und RAINER, Ingomar (Hrsg.). Kaiser Ferdinand III. Drama musicum (Wiener Edition alter Musik 43). Wien: Doblinger 2015.

KNAUS, Herwig. Die Musiker im Archivbestand des kaiserlichen Obersthofmeisteramtes (1637-1705) (Veröffentlichungen der Kommission für Musikforschung der Österreichischen Akademie der Wissenschaften 8), Band 2. Wien: Verlag der Österreichischen Akademie der Wissenschaften 1968.

[LEOPOLD WILHELM.] Diporti del Crescente. Bruxelles: Giovanni Mommartio1656.

MAREK, Pavel (ed). Svědectvi o ztrátě starého světa. Manželská korespondence Zdeňka Vojtěcha Popela z Lobkovic a Polyxeny Lobkovické z Pernštejna. České Budějovice: Historický ústav Jihočeské univerzity 2005.

SAUNDERS, Steven. Sacred Music at the Hapsburg Court of Ferdinand II (1615-1637): The Latin Vocal Works of Giovanni Priuli and Giovanni Valentini. Diss. University of Pittsburgh 1990/Ann Arbor: University Microfilms International.

SAUNDERS, Steven. The Emperor as Artist. New Discoveries Concerning Ferdinand III's Musical Compositions. In Studien zur Musikwissenschaft 45, 1996, S. 7-31.

SCHLAGER, Johann Evangelist. Über das alte Wiener Hoftheater In Sitzungsberichte der kaiserlichen Akademie der Wissenschaften. Philosophisch-historische Classe. 6. Bd. Wien: 1851, S. 147-171.

SCHREIBER, Renate. "Ein Galeria nach meinem Humor". Erzherzog Leopold Wilhelm. Wien: Kunsthistorisches Museum 2004.

SEIFERT, Herbert. Die Oper am Wiener Kaiserhof im 17. Jahrhundert (Wiener Veröffentlichungen zur Musikwissenschaft 25). Tutzing: Schneider 1985. 
SEIFERT, Herbert. Giovanni Sansoni, Cornettist, Maestro dei concerti and Composer in Venice, Graz and Vienna In De musica disserenda XIII/1-2, 2017, S. 147-156 (URL: https://ojs.zrc-sazu. si/dmd/article/view/6582/6234).

SEIFERT, Herbert. Kirchers Kompositionsschüler an den Habsburgerhöfen: Organisten und Kaiser In Melanie Wald-Fuhrmann (Hg.). Steinbruch oder Wissensgebäude? Zur Rezeption von Athanasius Kirchers Musurgia universalis in Musiktheorie und Kompositionspraxis (Bibliotheca Helvetica Romana XXXIV). Basel: Schwabe 2013, S. 131-141.

SEIFERT, Herbert. The Beginnings of Sacred Dramatic Musical Works at the Imperial Court of Vienna: Sacred and Moral Opera, Oratorio and Sepolcro In BESUTTI, Paola (ed.). L'Oratorio musicale italiano e $i$ suoi contesti (secc. XVII-XVIII) (Quaderni della Rivista italiana di musicologia 35), Firenze: Olschki 2002, S. 489-511; also in id. Texte zur Musikdramatik im 17. und 18. Jahrhundert. Aufsätze und Vorträge. Hrsg. v. Matthias J. Pernerstorfer (Summa Summarum 2), Wien: Hollitzer 2014, S. 765-782.

VALENTINI, Giovanni, Anagrammata supra nomen Jesum, Jesu, Jesus ante sanctam communionem, Wien: Matthaeus Cosmerovius 1649.

VALENTINI, Giovanni. Cento e trenta quattro anagrammi sovra il glorioso nome di Santa Caterina martire, con nove ottave obligate, Wien: Matthaeus Cosmerovius 1647.

VALENTINI, Giovanni. Cento e venti Anagrammi Sovra Santo Saverio Apostolo dell'Indie, Wien: Matthaeus Cosmerovius 1646.

WACKERNAGEL, Bettina. Bayerische Staatsbibliothek. Katalog der Musikhandschriften 3. Collectio musicalis Maximilianea. München: Henle 1981. 